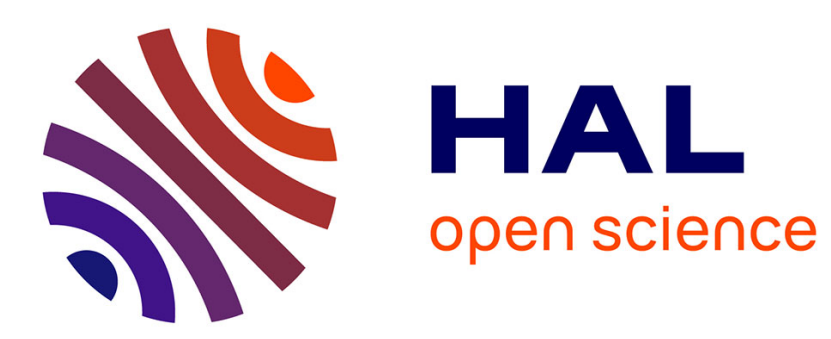

\title{
Control of a separated boundary layer: Reduced-order modeling using global modes revisited
}

Uwe Ehrenstein, Pierre-Yves Passaggia, Francois Gallaire

\section{To cite this version:}

Uwe Ehrenstein, Pierre-Yves Passaggia, Francois Gallaire. Control of a separated boundary layer: Reduced-order modeling using global modes revisited. Theoretical and Computational Fluid Dynamics, 2011, 25 (1), pp.195-207. 10.1007/s00162-010-0195-5 . hal-00433158

\section{HAL Id: hal-00433158 https://hal.science/hal-00433158}

Submitted on 18 Nov 2009

HAL is a multi-disciplinary open access archive for the deposit and dissemination of scientific research documents, whether they are published or not. The documents may come from teaching and research institutions in France or abroad, or from public or private research centers.
L'archive ouverte pluridisciplinaire HAL, est destinée au dépôt et à la diffusion de documents scientifiques de niveau recherche, publiés ou non, émanant des établissements d'enseignement et de recherche français ou étrangers, des laboratoires publics ou privés. 


\title{
Control of a separated boundary layer: Reduced-order modeling using global modes revisited
}

Received: date / Accepted: date

\begin{abstract}
The possibility of model reduction using global modes is readdressed, aiming at the controlling of a globally unstable separation bubble induced by a bump geometry. A combined oblique and orthogonal projection approach is proposed to design an estimator and controller in a Riccati-type feedback setting. An input-output criterion is used to appropriately select the modes of the projection basis. The full-state linear instability dynamics is shown to be successfully controlled by the feedback coupling with controllers of moderate degrees of freedom.
\end{abstract}

Keywords Separated boundary layer $\cdot$ model reduction $\cdot$ control

PACS 47.20.Ft $\cdot 47.20 . \mathrm{Ib} \cdot 47.85 . \mathrm{ld}$

\section{Introduction}

Separated boundary layer flows are ubiquitous in both external and internal practical flows, like the rear of a vehicle or wings at high incidence. They are often associated with strong instabilities with a broad frequency spectrum which are still not entirely understood, despite a longstanding theoretical, numerical and experimental effort (see [2], [18]). As an archetype configuration, the backward-facing step, showing various phenomena associated with flow separation, has been often considered for the purpose of control. Feedback and instantaneous control strategies have been developed for this flow case, for instance to reduce the recirculation bubble length in the laminar flow case [6] or to increase mixing in turbulent flow [12]. In the present paper, a recirculation region created by a boundary layer encountering a shallow bump is considered. For the same flow geometry the instability behavior has been analyzed recently in [9], using the numerical global instability setting. This flow configuration and the associated numerical model is appropriate for feedback control in the context of linear systems approach to flow control, which has found a lot of attention during the last decade (see the review $[13])$.

Given the extensive degrees of freedom of the flow dynamics, control theory can however hardly be applied to the dynamical system which governs the full flow state and the problem of model reduction is intimately related to the control issue. Very recently the input-output formulation of flow control problems has led to promising results with regard to model reduction and it has been successfully applied to the control of channel flow [11] or the flat-plate boundary layer [3]. The projection basis in

U. Ehrenstein

IRPHÉ UMR 6594, Aix-Marseille Université, CNRS, 49, Rue Joliot-Curie, F-13384 Marseille Cedex 13, France

P.-Y. Passaggia

IRPHÉ UMR 6594, Aix-Marseille Université, CNRS, 49, Rue Joliot-Curie, F-13384 Marseille Cedex 13, France

F. Gallaire

Laboratoire J. A. Dieudonné, Université de Nice-Sophia Antipolis, Parc Valrose, F-06108 Nice Cedex 02, France 
this approach is close to the optimal reduced-order model, with respect to observability and controllability for the specific actuators and sensors considered. Some attempts have also been made to exploit in the model reduction procedure the underlying physical mechanisms of the flow instability and in particular to use the global modes for projection. This approach has been applied to a shallow cavity [1] with some success. Very recently, the reliability of model reduction using global modes has been questioned in [5], considering an open square cavity. The performance of the resulting compensator obtained via bi-orthogonal projection for this non-normal flow case is shown in [5] to be less efficient, in comparison with models based on proper orthogonal decomposition and balanced modes. However, while the latter methods are designed to optimally reproduce the input-output behavior, they necessitate the computation and the approximation in a time-stepping procedure of the Gramians associated with controllability and observability [19].

In the present work, the possibility of model reduction using global mode subspaces is readdressed by focusing on an alternative orthogonal projection approach associated with a least-square optimization. The control and estimation are designed via Riccati-type feedback and the capability of the compensator to suppress the global perturbation dynamics of the separation bubble is assessed. The paper is organized as follows. In section 2 the flow model with the corresponding numerical large-scale system is provided. The control and estimation issues are introduced in section 3 and the reducedorder model is assessed by comparing bi-orthogonal and least-square projections onto a set of global modes. Reduced-order systems of different dimensions are designed, based on a selection criterion for the model basis. The ability of the compensator to control the full-state flow dynamics is demonstrated in section 4. Finally, some conclusions are drawn in section 5.

\section{Flow configuration and numerical model}

The flow geometry in dimensionless variables is $0 \leq x \leq L, \eta(x) \leq y \leq H$, with $\eta(x)$ the lower boundary containing a bump with height $h$. A boundary-layer flow evolves along the bump geometry and the displacement thickness $\delta^{*}$ of the Blasius profile $(U(y), 0)$, imposed at inflow $x=0$, has been chosen as reference length, the Reynolds number being $R e=\delta^{*} U_{\infty} / \nu$ with $U_{\infty}$ the uniform flow velocity far from the wall.

The same geometry has been used in [15],[9] and the basic flow state exhibits an elongated recirculation bubble at the rear of the bump (cf. figure 1 for a bump height $h=2$ ). The flow states at increasing Reynolds numbers have been computed as nonlinear equilibrium solutions of the stationary Navier-Stokes system

$\mathbf{f}(\mathbf{u}, p, R e)=\left[-(\mathbf{u} \cdot \nabla) \mathbf{u}-\nabla p+(1 / R e) \nabla^{2} \mathbf{u} ; \nabla \cdot \mathbf{u}\right]=\mathbf{0}$,

for the flow velocity $\mathbf{u}=(u, v)$ and pressure $p$. At outflow $x=L$, homogeneous Neumann boundary conditions for the flow velocity field $\mathbf{u}$ are prescribed, whereas at $y=H$ the upper boundary condition $\mathbf{u}=(1,0)$ is imposed, which is consistent with uniform flow conditions far outside the boundary layer. The system has been discretized using Chebyshev collocation in both the streamwise $x$ direction and wall-normal $y$ direction, together with an algebraic mapping transforming the physical domain into the Cartesian computational domain $[-1,1] \times[-1,1]$. To recover a steady equilibrium state, a quasiNewton method together with an arclength continuation procedure has been used. Details about the solution procedure are provided in [9].

In figure 1, equilibrium states at $R e=590,690$ are depicted and the recirculation length is seen to increase with the Reynolds number. A critical issue is the appropriate choice of the length and height of the computational domain, in particular when addressing the stability by computing global modes spectra of the steady states. In [9] it has been shown that the overall instability dynamics is reproduced within a range of the parameter values, although definite convergence of the eigenvalue spectrum can hardly be achieved for the present highly non-normal flow case (see for instance [20] for a general discussion of non-normal operators). Indeed, the flow dynamics depends on the mutual interaction of the modes, rather than the individual eigenvalue locations inside the spectrum. The reference case in [9] has been computed for $L=300, H=30$, for a grid with $N_{x}=250$ collocation points in $x$ and $N_{y}=40$ collocation points in $y$. These values have been chosen in the present analysis.

In the following we denote

$\mathbf{A}\left(\mathbf{u}_{s}, R e\right)=D_{(\mathbf{u}, p)} \mathbf{f}\left(\mathbf{u}_{s}, p_{s}, R e\right)$ 
(a)

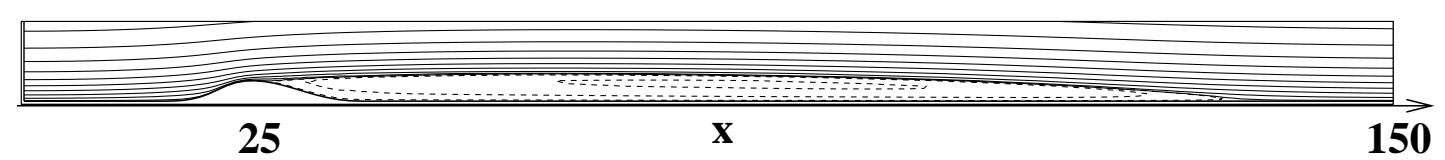

(b)

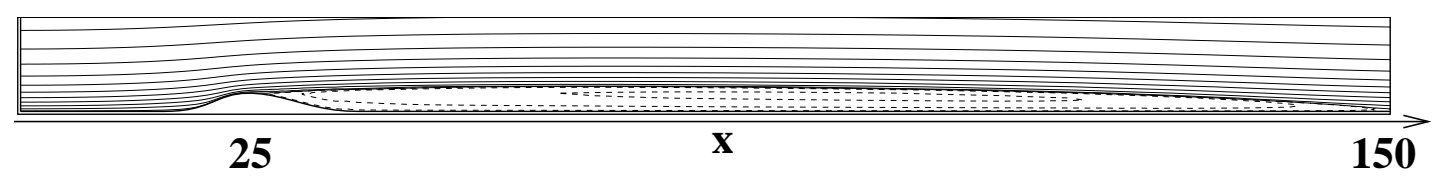

Fig. 1 Streamlines in the vicinity of the wall boundary of steady flow state at (a) $R=590$, (b) $R e=690$, for a bump height $h=2$.

the Jacobian matrix evaluated at basic equilibrium states $\left(\mathbf{u}_{s}, p_{s}\right)$. The dynamics of linear disturbances is hence governed by the system

$\mathbf{E} \frac{d}{d t} \mathbf{q}=\mathbf{A}\left(\mathbf{u}_{s}, R e\right) \mathbf{q}, \quad \mathbf{E} \mathbf{q}=(u, v, 0)$,

with $\mathbf{q}=(u, v, p)$ the linear flow disturbance. The disturbance flow velocity satisfies the no-slip condition at the wall and is set to zero at inflow $x=0$ and at the upper boundary $y=H$, where uniform basic flow is retrieved. At outflow, homogeneous Neumann boundary conditions for the perturbation flow velocity are prescribed. The vector $\mathbf{q}$ contains the discretized flow field as well as the pressure which gives rise to the differential-algebraic equations (3). The pressure is given implicitly through the incompressibility condition which is imposed in the interior domain as well as on the boundary. Spurious pressure modes are inherent when a Chebyshev discretization is used in a rectangular box [17]. Continuity of the wall-normal pressure gradient is imposed at the four corners and the four remaining spurious pressure modes, that is the arbitrary constant and those arising from the Chebyshev disretization (cf. page 262 in [17]), are eliminated through simple algebraic relations, similar to the procedure outlined in [8]. The matrix $\mathbf{A}\left(\mathbf{u}_{s}, R e\right)$ is hence non singular and $\mathbf{E}$ being the projection of the solution vector $\mathbf{q}$ onto the perturbation flow velocity components, system (3) may be integrated using multistep backward differentiation formulae (BDF), which are appropriate for the time-integration of differential-algebraic systems [14].

The stability of the basic steady state $\left(\mathbf{u}_{s}, p_{s}\right)$ is computed by considering the two-dimensional temporal modes

$\mathbf{q}=\widehat{\mathbf{q}}(x, y) e^{-i \omega t}, \quad \widehat{\mathbf{q}}=(\widehat{u}, \widehat{v}, \widehat{p})$

and hence according to (3) the eigenvalues $\omega_{j}$ and eigenvectors $\widehat{\mathbf{q}}_{j}$ are solution of

$-i \omega_{j} \mathbf{E} \widehat{\mathbf{q}}_{j}=\mathbf{A} \widehat{\mathbf{q}}_{j}$

with $\mathbf{A}$ the Jacobian matrix (we omit the reference to the basis state $\mathbf{u}_{s}$ and Reynolds number). The resulting large eigenvalue problem is solved using large-scale Krylov subspace projections together with the Arnoldi algorithm and the stability for the recirculation bubble has been assessed in [9].

The unstable part of the spectrum, is depicted in figure 2 , for $R e=590,690$, which shows the drastic increase of the amplification rates $\omega_{i}>0$ when increasing the Reynolds numbers. The Reynolds number $R e=590$, for a bump height $h=2$ is slightly above the threshold for the global dynamics analyzed in [9]. In this latter work the low-frequency oscillations of the recirculation bubble, which had been observed in direct numerical simulations [15], has been interpreted in terms of interaction of global modes.

Here, the goal is to evaluate the possibility of feedback control of the underlying global instability dynamics.

\section{Control, estimation and modal decomposition}

The possibility of model reduction, using global modes, in view of feedback flow control has been explored recently, for incompressible cavity flows considering a smooth shallow geometry in [1] or a 


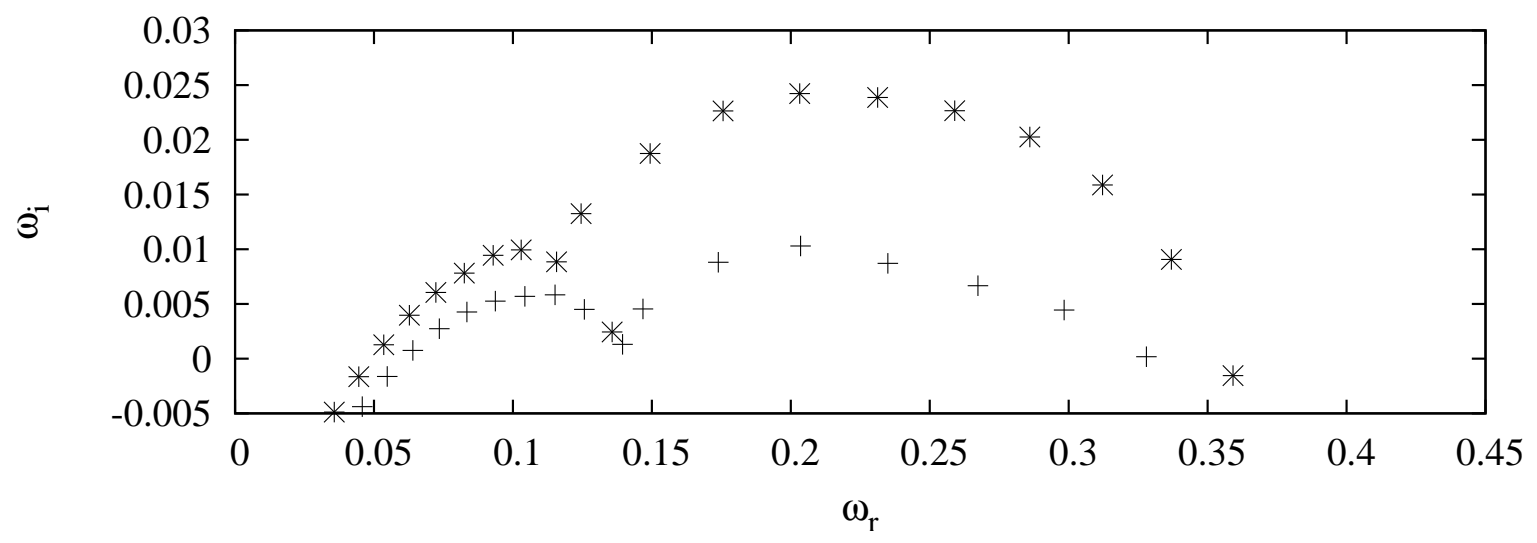

Fig. 2 Unstable part of the spectrum ; +: $R e=590$ (a) $R=590$, (b) $R e=690$, for a bump height $h=2$.

(a)

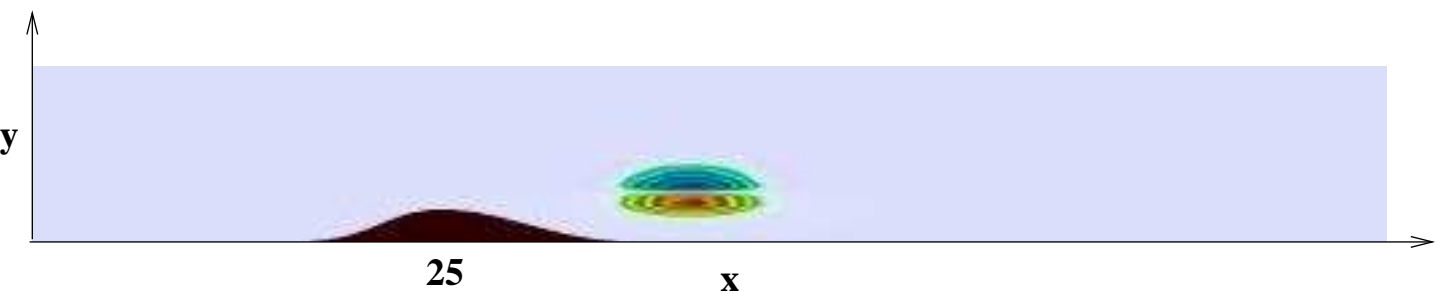

(b)

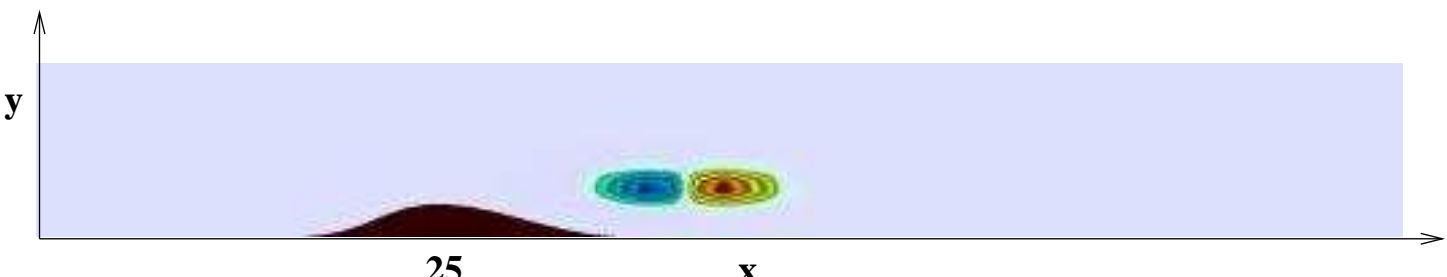

25

$\mathbf{X}$

Fig. 3 Actuator, (a) streamwise component $B_{x}$ and (b) wall-normal component $B_{y}$.

sharp-edge geometry in [4]. Given the degrees of freedom of real full flow states, only a partial-state control approach is feasible in general and hence the flow state has to be estimated. A control device has to be designed which efficiently acts on the flow dynamics and sensors are implemented to measure significant flow quantities.

In the present work, which aims at reassessing model reduction using global modes rather than providing realistic flow control, a divergence-free spatially localized volume force $\mathbf{B}=\left(B_{x}, B_{y}\right)$ has been chosen as actuator. The actuator has a Gaussian envelope

$$
\begin{aligned}
& B_{x}=-\left(y-y_{0}\right) \exp \left(-\frac{\left(x-x_{0}\right)^{2}}{2 \sigma_{x}^{2}}-\frac{\left(y-y_{0}\right)^{2}}{2 \sigma_{y}^{2}}\right), \\
& B_{y}=\frac{\sigma_{y}^{2}}{\sigma_{x}^{2}}\left(x-x_{0}\right) \exp \left(-\frac{\left(x-x_{0}\right)^{2}}{2 \sigma_{x}^{2}}-\frac{\left(y-y_{0}\right)^{2}}{2 \sigma_{y}^{2}}\right) .
\end{aligned}
$$

The parameters $\left(x_{0}, y_{0}\right)$ fix the location of the actuator in space and $\left(\sigma_{x}, \sigma_{y}\right)$ accounts for its spatial extent. Actuation in the vicinity of the bump is expected to provide efficient response, according to the shape of the adjoint eigenvectors associated with the most unstable direct modes, one of which being shown in figure 5 (cf. section 3.1). We have chosen $x_{0}=40$, slightly downstream of the bump, and $y_{0}=3$. The values $\sigma_{x}=2, \sigma_{y}=0.5$ give rise to an actuator $\mathbf{B}$ depicted in figure 3 , with a width and height of approximately 10 and 1.5 units, respectively. 
The sensors are placed at several locations along the plate, starting approximately at the center of the steady flow recirculation bubble and extending to the outflow region of the computational domain. They measure the flow skin-friction and the measurements are collected in a vector $\mathbf{y}$ with components

$y_{i}=\int_{x_{i}}^{x_{i}+\Delta x} \frac{\partial u}{\partial y}(x, 0) d x, \quad i=1, \cdots 6$,

where $\Delta x=20$ and $x_{1}=60, x_{2}=100, x_{3}=140, x_{4}=180, x_{5}=220, x_{6}=260$.

In the absence of the full knowledge of the state vector $\mathbf{q}$, a state estimate $\mathbf{q}_{e}$ is introduced, which mimics the unknown state vector: It is governed by the same dynamics, but also forced by a restoring term $\mathbf{L}\left(\mathbf{y}_{e}-\mathbf{y}\right)$ for the real measurements to be followed as exactly as possible. In essence, the estimator receives the measurements, determines the estimated flow which is fed back by the controller. Adding the actuator $\mathbf{B}$ to system (3) and introducing a measurement operator $\mathbf{C}$, the coupling with the estimator and the controller gives rise to the system

$$
\begin{aligned}
\mathbf{E} \frac{d}{d t} \mathbf{q} & =\mathbf{A q}+\mathbf{B} \phi, \quad \mathbf{y}=\mathbf{C q}, \\
\mathbf{E} \frac{d}{d t} \mathbf{q}_{e} & =\mathbf{A} \mathbf{q}_{e}+\mathbf{B}_{e} \phi+\mathbf{L}\left(\mathbf{y}_{e}-\mathbf{y}\right), \quad \mathbf{y}_{e}=\mathbf{C} \mathbf{q}_{e}, \quad \phi=\mathbf{K} \mathbf{q}_{e} .
\end{aligned}
$$

The aim of the control design is to determine the operators $\mathbf{K}, \mathbf{L}$, which are called respectively control gain and estimation gain (see the review [13] and the references therein). The dynamical system (10), called the compensator, provides feedback control $\phi$ when receiving the flow measurement $\mathbf{y}$. At this stage, note that we are free to choose $\mathbf{B}_{e}$ as a model of $\mathbf{B}$, although $\mathbf{B}=\mathbf{B}_{e}$ is used in many studies. Only the final coupling of the compensator (10) and the plant (9) will assess the quality of this modeling. For systems of large size, $\mathbf{K}$ and $\mathbf{L}$ cannot be determined without resorting to a model reduction procedure. In our approach we decide to reduce the dynamics of the estimated flow state on the invariant subspace of a set of eigenmodes, solution of the generalized eigenvalue problem (5).

The operators being real, the eigenvectors are complex conjugate pairs and

$\mathbf{B}_{e}=\mathbf{E} \sum_{i=1}^{m}\left(\beta_{j} \widehat{\mathbf{q}}_{j}+\bar{\beta}_{j} \overline{\mathbf{q}}_{j}\right)$

(the variables with the bar being the complex conjugate) with $\widehat{\mathbf{q}}_{j}$ solutions of (5). The operator $\mathbf{B}_{e}$ may equivalently be written

$\mathbf{B}_{e}=\mathbf{E V} \widehat{\mathbf{B}}$

for the real vector $\widehat{\mathbf{B}}=\left(2 \beta_{1 r},-2 \beta_{1 i}, \cdots, 2 \beta_{m r},-2 \beta_{m i}\right)^{T}$ (with $\left.\beta_{j}=\beta_{j r}+i \beta_{j i}\right)$ and the real matrix

$\mathbf{V}=\left(\widehat{\mathbf{q}}_{1 r} \widehat{\mathbf{q}}_{1 i} \cdots \widehat{\mathbf{q}}_{m r} \widehat{\mathbf{q}}_{m i}\right)$,

the $n=2 m$ columns of which being the real parts $\widehat{\mathbf{q}}_{j r}$ and imaginary parts $\widehat{\mathbf{q}}_{j r}$ of the eigenvectors (for corresponding eigenvalues $\lambda_{j}=-i \omega_{j}$ with non-zero imaginary parts). The estimated flow state is expanded into the basis of eigenvectors as well, with

$\mathbf{q}_{e}=\mathbf{V} \widehat{\mathbf{x}}_{e}$

and the compensator (10) can be written equivalently as the $n$-dimensional system

$\frac{d}{d t} \widehat{\mathbf{x}}_{e}=\boldsymbol{\Lambda} \widehat{\mathbf{x}}_{e}+\widehat{\mathbf{B}} \phi+\widehat{\mathbf{L}}\left(\mathbf{y}_{e}-\mathbf{y}\right), \quad \mathbf{y}_{e}=\widehat{\mathbf{C}} \widehat{\mathbf{x}}_{e}, \quad \phi=\widehat{\mathbf{K}} \widehat{\mathbf{x}}_{e}$

where $\widehat{\mathbf{C}}=\mathbf{C V}$. Note that in this real vectorspace formulation, the matrix $\boldsymbol{\Lambda}$ is not diagonal but has an evident block-like structure with $2 \times 2$ blocks centered at its diagonal of the form

$$
\left(\begin{array}{cc}
\omega_{j i} & -\omega_{j r} \\
\omega_{j r} & \omega_{j i}
\end{array}\right)
$$

with $\lambda_{j}=-i \omega_{j}=\omega_{j i}-i \omega_{j r}$ the eigenvalues solution of $(5)$.

Before addressing the projection issue, we briefly outline the main steps to compute the coefficients $\widehat{\mathbf{K}}$ and $\widehat{\mathbf{L}}$ of the control gain and estimation gain. Considering the flow dynamics within the subspace spanned by the chosen set of modes, the reduced state vector is $\mathbf{q}_{r}=\mathbf{V} \widehat{\mathbf{x}}$ with $\widehat{\mathbf{x}}$ solution of

$\frac{d}{d t} \widehat{\mathbf{x}}=\boldsymbol{\Lambda} \widehat{\mathbf{x}}+\widehat{\mathbf{B}} \phi, \quad \phi=\widehat{\mathbf{K}} \widehat{\mathbf{x}}$ 
In order to determine an optimal control gain $\widehat{\mathbf{K}}$, the functional

$\mathcal{J}=\frac{1}{2} \int_{0}^{T} \widehat{\mathbf{x}}^{T} \mathbf{V}^{T} \mathbf{W} \mathbf{V} \widehat{\mathbf{x}} d t+\frac{l^{2}}{2} \int_{0}^{T} \phi^{2} d t$,

is minimized, the first term of the right-hand side being the projected flow energy, with $\mathbf{W}$ a diagonal matrix with the appropriate weights for the numerical quadrature of the integral $\int\left(u^{2}+v^{2}\right) d x d y$ in the flow domain. The parameter $l^{2}$ puts a bound on the control cost and the value $l^{2}=10^{8}$ has been taken in the present computations. For time-horizon $T \rightarrow \infty$, the control gain $\widehat{\mathbf{K}}$ can be obtained as $\widehat{\mathbf{K}}=-l^{-2} \widehat{\mathbf{B}} \mathbf{X}_{c}$, the matrix $\mathbf{X}_{c}$ being solution of an algebraic Riccati equation. Details are not provided here, the procedure being outlined in the review [13] and it has been used in [4], [3], [1], among others. The reduced estimation error $\widetilde{\mathbf{x}}=\widehat{\mathbf{x}}-\widehat{\mathbf{x}}_{e}$ is solution of

$\frac{d}{d t} \widetilde{\mathbf{x}}=\mathbf{\Lambda} \widetilde{\mathbf{x}}+\widehat{\mathbf{L}} \widetilde{\mathbf{y}}, \quad \widetilde{\mathbf{y}}=\widehat{\mathbf{C}} \widetilde{\mathbf{x}}$

As shown in [13], p. 399, there is a formal analogy between (18) and (16) and the estimation gain $\widehat{\mathbf{L}}$ can be written $\widehat{\mathbf{L}}=-l_{e}^{-2} \mathbf{X}_{e} \widehat{\mathbf{C}}^{T}$ with $\mathbf{X}_{e}$ solution of the Riccati equation

$$
\boldsymbol{\Lambda} \mathbf{X}_{e}+\mathbf{X}_{e} \boldsymbol{\Lambda}^{T}-l_{e}^{-2} \mathbf{X}_{e} \widehat{\mathbf{C}}^{T} \widehat{\mathbf{C}} \mathbf{X}_{e}+\mathbf{Q}=0
$$

The estimation gain is also called Kalman filter and in a stochastic setting the parameter $l_{e}^{-2}$ can be interpreted as a sensor noise variance, whereas the matrix $\mathbf{Q}$ in the above equation corresponds to the covariance matrix of modeled white state noise [10]. No particular disturbance and measurement noise modeling has been performed here and it is convenient to chose $\mathbf{Q}=\mathbf{I}$ (cf. [13]), whereas the value $l_{e}^{2}=10^{8}$ enforces low-amplitude feedback gain.

\subsection{Bi-orthogonal and least-square projection}

The conventional projection procedure for non-normal problems uses the bi-orthogonal property

$<\widehat{\mathbf{q}}_{k}^{+}, \mathbf{E} \widehat{\mathbf{q}}_{j}>=\delta_{k j}$

with respect to the Hermitian inner product, the adjoint modes $\hat{\mathbf{q}}_{k}^{+}$being solution of the generalized eigenvalue problem

$i \omega_{k} \mathbf{E} \widehat{\mathbf{q}}_{k}^{+}=\mathbf{A}^{T} \widehat{\mathbf{q}}_{k}^{+}$

of the adjoint system with $\mathbf{A}^{T}$ merely the transposed discretized operator. In order to address the coupling between the large scale plant (9) and the low-dimensional compensator (15), it is convenient to consider the compensator written in physical space (10), the systems (10) and (15) being equivalent. Indeed, by making use of the bi-orthogonal property, the expansion (14) may be written equivalently as

$$
\mathbf{q}_{e}=\sum_{i=1}^{m}\left(\alpha_{j} \widehat{\mathbf{q}}_{j}+\bar{\alpha}_{j} \overline{\mathbf{q}}_{j}\right), \quad \text { with } \quad \alpha_{j}=<\widehat{\mathbf{q}}_{j}^{+}, \mathbf{E} \mathbf{q}_{e}>,
$$

the $n=2 m$ coefficients of $\widehat{\mathbf{x}}_{e}$ in (14) being given by the real and imaginary parts of $\alpha_{j}$ with $\widehat{x}_{e, 2 j-1}=$ $2 \alpha_{j r}, \widehat{x}_{e, 2 j}=-2 \alpha_{j i}, j=1, \cdots, m$. Hence, one may write $\widehat{\mathbf{x}}_{e}=\mathbf{P q}_{e}$ for some operator $\mathbf{P}$ and hence the control and estimation gains in (10) and (15) are connected through the relations

$$
\mathbf{K}=\widehat{\mathbf{K}} \mathbf{P}, \quad \mathbf{L}=\mathbf{V} \widehat{\mathbf{L}}
$$

with $\mathbf{V}$ defined by (13). Subtracting (10) from (9), the error $\widetilde{\mathbf{q}}=\mathbf{q}-\mathbf{q}_{e}$ between the real and the estimated flow state is solution of

$\mathbf{E} \frac{d}{d t} \widetilde{\mathbf{q}}=\mathbf{A} \widetilde{\mathbf{q}}+\left(\mathbf{B}-\mathbf{B}_{e}\right) \phi+\mathbf{L} \widetilde{\mathbf{y}}, \quad \widetilde{\mathbf{y}}=\mathbf{C} \widetilde{\mathbf{q}}$.

The actuation error

$\widetilde{\mathbf{B}}=\mathbf{B}-\mathbf{B}_{e}$ 
depends on the specific expansion coefficients in (11). In the bi-orthogonal projection approach, these coefficients are provided by the inner products between the adjoint modes and the real actuator and

$$
\beta_{j}=<\widehat{\mathbf{q}}_{j}^{+}, \mathbf{E B}>, \quad j=1, \cdots, m
$$

This procedure has for instance been used in [1] and it ensures that the actuation error $\widetilde{\mathbf{B}}$ has no contributions with respect to the chosen modal subspace. However, as for instance discussed in [5], there is no guarantee that the error does not excite flow states outside the range of the reduced order model.

This appears to be a major difficulty in this a priori model reduction approaches, in contrast to the input-output analysis for flow control based on observability and controllability operators. In this latter approach, which has recently been applied for instance to the control of channel flow in [11] or the flat-plate boundary layer in [3], the actuator $\mathbf{B}$ enters directly into the construction of the so-called balanced modes used for reduction.

At this stage, the choice of the direction of projection (either orthogonal or oblique) remains open. In the present flow case, there are unstable modes, depicted in figure 2, and it appears to be mandatory to use bi-orthogonal projection for the unstable part of the spectrum. Indeed, it is unlikely that a control may be achieved, if the actuation error (22) has contributions with regard to unstable modes. However, concerning the stable part of the spectrum, alternative projection approaches are possible.

The matrix (13) contains the real and imaginary parts of the set of eigenvectors and we separate the block with the $m_{u}$ unstable eigenvectors from the block containing the $m_{s}$ stable ones, by writing

$$
\mathbf{V}=\left(\mathbf{V}_{u} \mathbf{V}_{s}\right)
$$

The expansion coefficients of $\mathbf{B}_{e}$ with respect to the unstable eigenvectors are first determined by bi-orthogonal projection of $\mathbf{B}$, i.e. $\beta_{j}=<\widehat{\mathbf{q}}_{j}^{+}, \mathbf{E B}>, \quad j=1, \cdots, m_{u}$, giving rise to

$\mathbf{B}_{u}=\mathbf{E} \mathbf{V}_{u} \widehat{\mathbf{B}}_{u}$.

Note that $m_{u}=15$ for the flow state at $R e=590$ and $m u=17$ for $R e=690$. Writing

$\mathbf{B}_{e}=\mathbf{B}_{u}+\mathbf{B}_{s}$

the projection on the stable part of the spectrum $\mathbf{B}_{s}=\mathbf{V}_{s} \widehat{\mathbf{B}}_{s}$ is computed as solution of the least-square projection, which minimizes the energy norm of the actuation error

$\left\|\mathbf{V}_{s} \widehat{\mathbf{B}}_{s}-\left(\mathbf{B}-\mathbf{B}_{u}\right)\right\|^{2}=\min _{\widehat{y}}\left\|\mathbf{V}_{s} \widehat{y}-\left(\mathbf{B}-\mathbf{B}_{u}\right)\right\|^{2}$.

(Note that the norm $\|\mathbf{g}\|^{2}=\mathbf{g}^{T} \mathbf{W} \mathbf{g}$ is the discrete version of the domain integral $\int\left(g_{x}^{2}+g_{y}^{2}\right) d x d y$ for any volume function $g=\left(g_{x}, g_{y}\right)$.) Solving the associated normal equation

$\mathbf{V}_{s}^{T} \mathbf{W} \mathbf{V}_{s} \widehat{\mathbf{B}}_{s}=\mathbf{V}_{s}^{T} \mathbf{W}\left(\mathbf{B}-\mathbf{B}_{u}\right)$

one recovers the solution $\widehat{\mathbf{B}}_{s}$ of this optimization procedure. With the decomposition (24) the projection error (22) is orthogonal to the set of stable eigenmodes for the modal subspace considered and it has no contribution with respect to the unstable eigenmodes. The reduced actuator $\mathbf{B}_{e}$ is hence determined, within the chosen set of eigenvectors, such that it fits optimally the real actuator in the energy norm measure.

\subsection{Projection results and selection of modal basis}

The choice of the stable subspace is subject to some arbitrariness. Given the dimension of the large scale dynamical system (of order 30,000), Krylov-subspace projection are to be performed to compute parts of the spectrum. Performing several shifts in the nowadays widely used Arnoldi algorithm (cf. [16]), large parts of the spectrum have been computed. For each shift, a $L U$ decomposition of the system matrix is performed to compute the Krylov subspace. Note that we consider rather large Krylov subspaces, of dimension 1600, and five to six shifts had to be performed to recover the part of the spectrum shown in figure 4. Exploring the complex frequency plane, 1000 modes in the half plane $\omega_{r}>0$ are depicted in 4 , for the basic state at $R e=590$. There are unstable eigenvalues with 


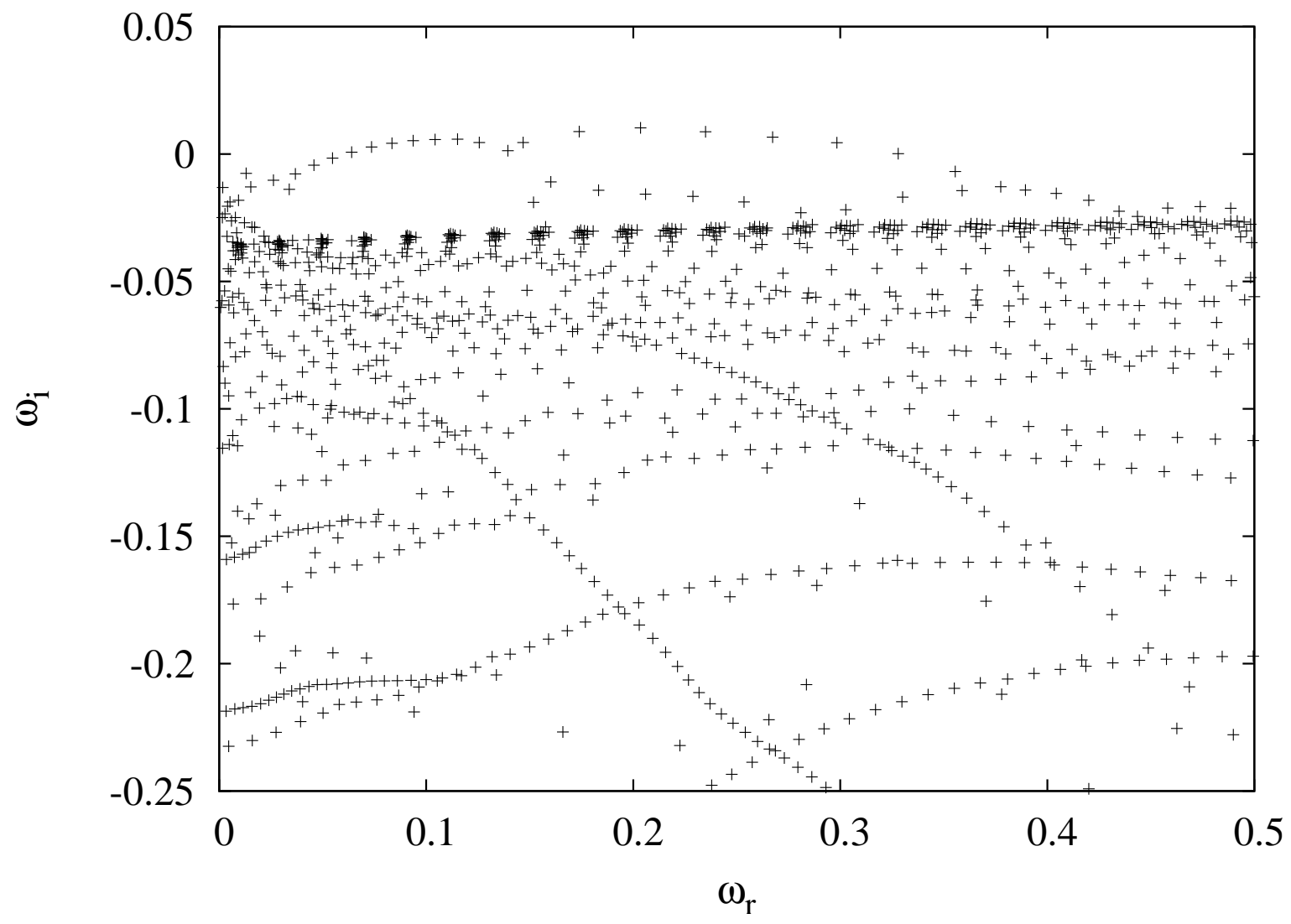

Fig. 4 Spectrum in the half plane $\omega_{r}>0$ with $m=1000$ eigenvalues $(R e=590)$.

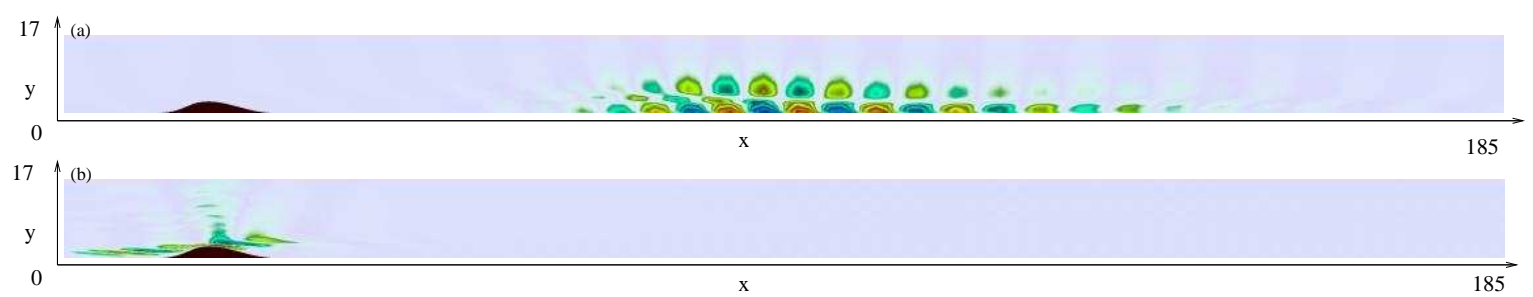

Fig. 5 (a) Streamwise component of the most unstable eigenmode at $\omega_{r} \approx 0.2$, cf. figure 2. (b) Streamwise component of the associated adjoint eigenfunction.

$\omega_{i}>0$ already shown in figure 2. Again, it has to be emphasized that we did not attempt to recover a definitely converged spectrum, which would hardly be feasible given the high non-normality of the operator in the large-scale system (3), which makes the precise eigenvalue locations sensitive to the grid-values used. The most unstable eigenmode (at $\omega_{r} \approx 0.2$, cf. figure 2 ) is depicted in figure 5(a) whereas the associated adjoint eigenvector structure is given in figure 5(b). As discussed for instance in [7], the clear separation in space between the direct and adjoint eigenfunction is associated with the strong streamwise non-normality.

The bi-orthogonal projection of the actuator onto the space spanned by the 1000 eigenvectors has been performed and the projected actuator in physical space is depicted in figure 6 (a). The projected volume forcing structure is very different from the real actuator shown in figure 3, the non-zero contributions visible downstream being precisely due to the strong non-normality. The adjoint modes are localized upstream, providing nonzero inner-products with the actuator, whereas the associated dominant direct modes are located downstream. 


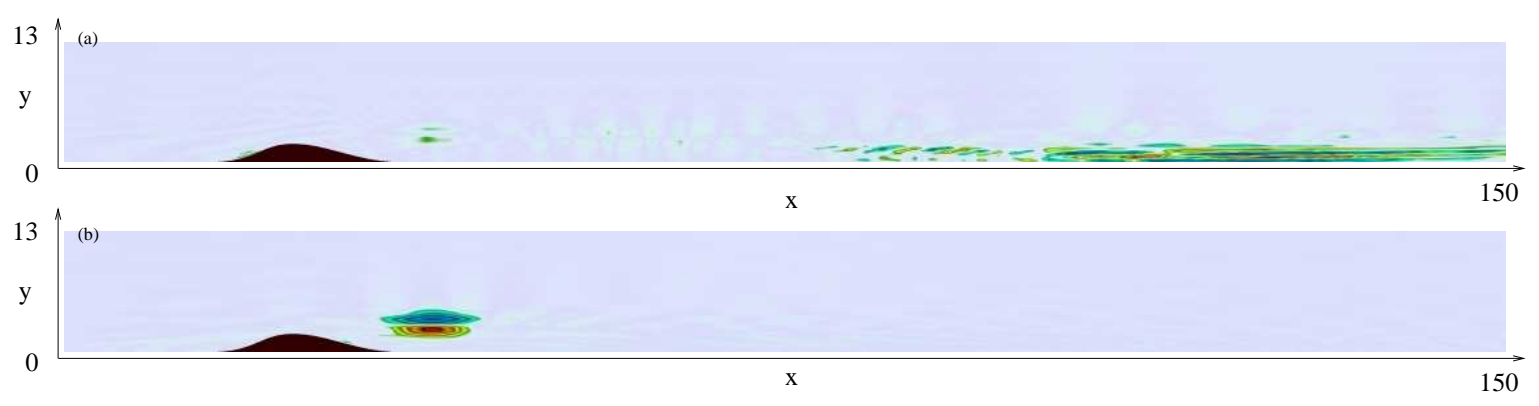

Fig. 6 Streamwise component $B_{x}$ of the projected actuator for the modal basis at $R e=590$; (a) bi-orthogonal projection, $m=1000$; (b) least-square projection, $m_{u}=15, m_{s}=985$.

By performing however the combined bi-orthogonal and least-square projection in the decomposition (24), one recovers the projected actuator depicted in figure 6 (b) which is seen to be quite close to the physical one. It would however be hazardous to infer merely from the reduced actuator structure, that the alternative projection works better than the conventional bi-orthogonal procedure. The performance of both approaches will be assessed in the next section.

The projection has been performed considering $m=1000$ complex eigenvectors and the associated reduced system in the real vectorspace (15) is of dimension $n=2 m=2000$. In the aim of realizing an efficient feedback with the plant, one would like the dimension of the compensator to be as small as possible and some criterion has to be applied to assess the amount of information contained in the modes, with regard to the control issue. This point has been addressed for instance in [3] and [5]. The idea is to quantify the output $\mathbf{y}=\mathbf{C q}$ for harmonic actuation inputs $\phi=e^{i \omega t}$ for the (complex) system

$\mathbf{E} \frac{d}{d t} \mathbf{q}=\mathbf{A q}+\mathbf{B}_{j} e^{i \omega t}$

and consequently

$\mathbf{y}=\mathbf{C}(i \omega \mathbf{E}-\mathbf{A})^{-1} \mathbf{B}_{j} e^{i \omega t}$,

with $\mathbf{B}_{j}$ the component of the projected actuator with respect to the eigenvector $\mathbf{q}_{j}$. The expansion in terms of the complex eigenvectors (together with the complex conjugate) is (11) and for $\mathbf{B}_{j}=\beta_{j} \mathbf{E} \widehat{\mathbf{q}}_{j}$, one gets

$\mathbf{y}=\mathbf{C} \widehat{\mathbf{q}}_{j}\left(\frac{1}{i \omega-\lambda_{j}}\right) \beta_{j} e^{i \omega t}$

with $\lambda_{j}=-i \omega_{j}$ the eigenvalue with eigenvector $\widehat{\mathbf{q}}_{j}$. The quantity $\mathbf{C} \widehat{\mathbf{q}}_{j}$ provides the skin friction measurements (8) of the global mode and is hence a vector $\mathbf{C}_{j}$ with 6 (complex) components, the amount of which indicating the observability of the mode, whereas $\beta_{j}$ is a measure of its controllability. From equation (29) it follows

$|\mathbf{y}| \leq \frac{\left|\mathbf{C}_{j}\right|\left|\beta_{j}\right|}{\left|\mathcal{R} e\left(\lambda_{j}\right)\right|}=\Gamma_{j}$

and the quantity $\Gamma_{j}$ is convenient to provide the hierarchy of the modes with respect to controllability and observability $\left(\left|\mathbf{C}_{j}\right|\right.$ is here the sum of the modulus of its 6 components). Note that $\left|\mathcal{R} e\left(\lambda_{j}\right)\right|=$ $\left|\mathcal{I} m\left(\omega_{j}\right)\right|$ and this quantity hence increases with the damping rate of the stable modes.

The $\Gamma_{j}$ values have been computed for the projected actuators for all the eigenvalues depicted in figure 4. The modes have been ordered for decreasing $\Gamma_{j}$ and for instance the 200 first modes with respect to this criterion are shown in figure $7(\mathrm{a})$, whereas the 50 first modes are depicted in figure 7 (b). The results at $R e=590$ are shown, for the coefficients of the actuator expansion obtained by the combined bi-orthogonal and least-square projection. First, the coefficients are computed for the highest $m_{\max }=1000$ value considered here and the criterion is applied to select a lower dimension $m$. The projection onto the stable subspace being orthogonal, the coefficients have then to be recomputed according to the least-square procedure. It has been checked that the procedure is hardly sensitive to the step $\Delta m$ used when diminishing the dimensions throughout this procedure. The general trend is that 

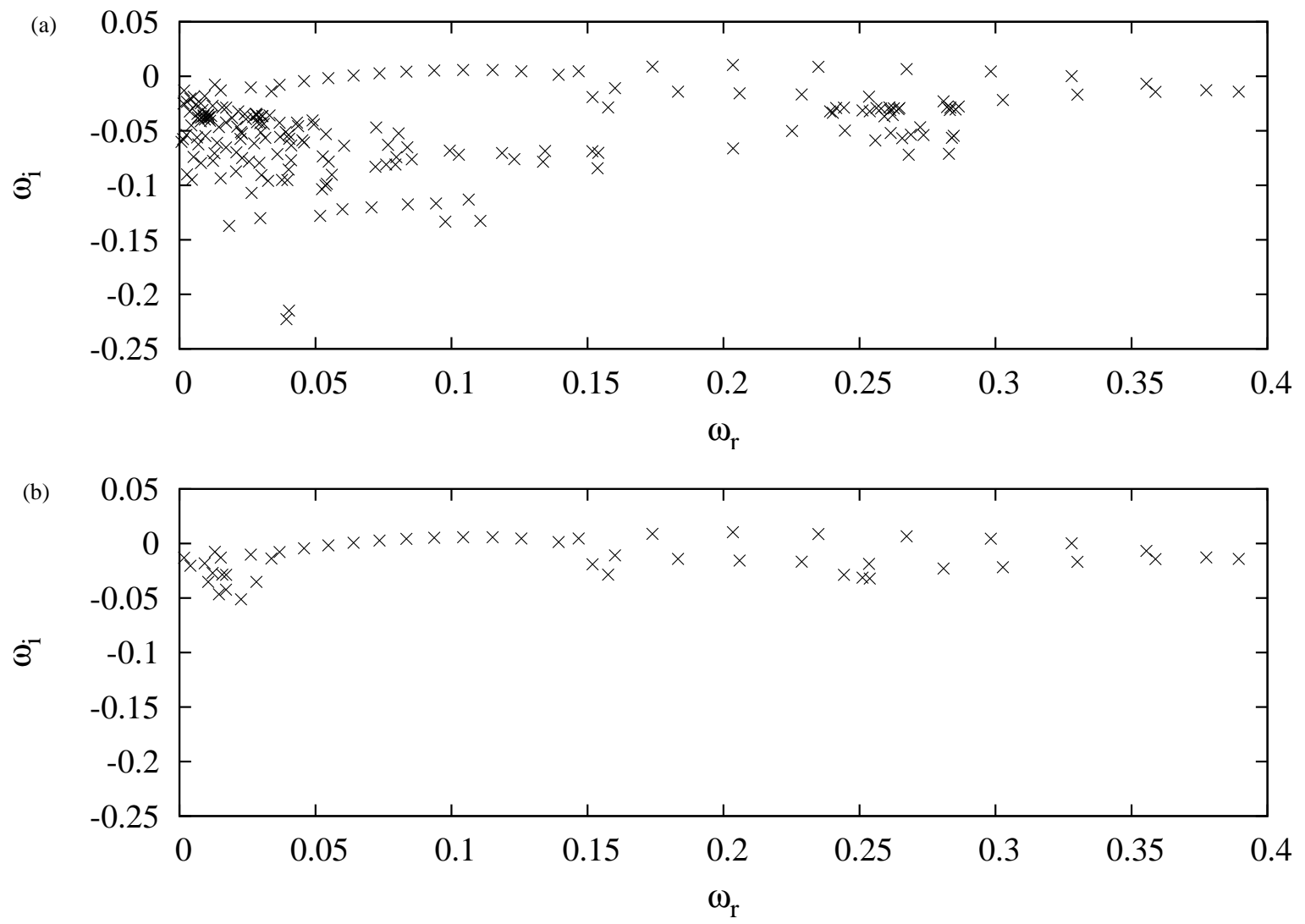

Fig. 7 Eigenvalues associated with decreasing $\Gamma_{j}$-values (flow case at $R e=590$ ); (a) 200 eigenvalues; (b) 50 eigenvalues.

for increasing damping rates (that is for decreasing imaginary parts of $\omega_{j}$ ), the $\Gamma_{j}$ values decrease. As already observed by [5], there is however some evidence that a simple selection criterion based only on the damping rates of modes may not be reliable. For instance, the spectrum with $m=200$ eigenvalues shown in figure 7 (a) still contains eigenvalues with relatively high damping rates.

The criterion has also been applied to the reduced actuator obtained by conventional bi-orthogonal projection. For a given dimension, the selected basis, not shown here, appeared to be essentially the same (with however different coefficients in the actuator expansion).

\section{Control results}

Putting together the plant (9) and the compensator (15), one recovers the system

$$
\begin{aligned}
\mathbf{E} \frac{d}{d t} \mathbf{q} & =\mathbf{A q}+\mathbf{B} \phi, \quad \mathbf{y}=\mathbf{C q} \\
\frac{d}{d t} \widehat{\mathbf{x}}_{e} & =(\boldsymbol{\Lambda}+\widehat{\mathbf{B}} \widehat{\mathbf{K}}+\widehat{\mathbf{L}} \widehat{\mathbf{C}}) \widehat{\mathbf{x}}_{e}-\widehat{\mathbf{L}} \mathbf{y} \\
\phi & =\widehat{\mathbf{K}} \widehat{\mathbf{x}}_{e} .
\end{aligned}
$$

The second-order backward differentiation formula (BDF) has been used for the integration of the differential-algebraic system $(31)$, and the vector $\mathbf{q}^{n+1}$ at time $t_{n+1}=(n+1) \Delta t$ is solution of

$$
\left(\frac{3}{2 \Delta t} \mathbf{E}-\mathbf{A}\right) \mathbf{q}^{n+1}=\frac{1}{2 \Delta t} \mathbf{E}\left(4 \mathbf{q}^{n}-\mathbf{q}^{n-1}\right)+\mathbf{B} \phi^{n+1} \text {. }
$$

Even for large and full matrices, as arising from the present Chebyshev-collocation approximation, the $L U$ decomposition of the left-hand side can be performed with nowadays available computers 


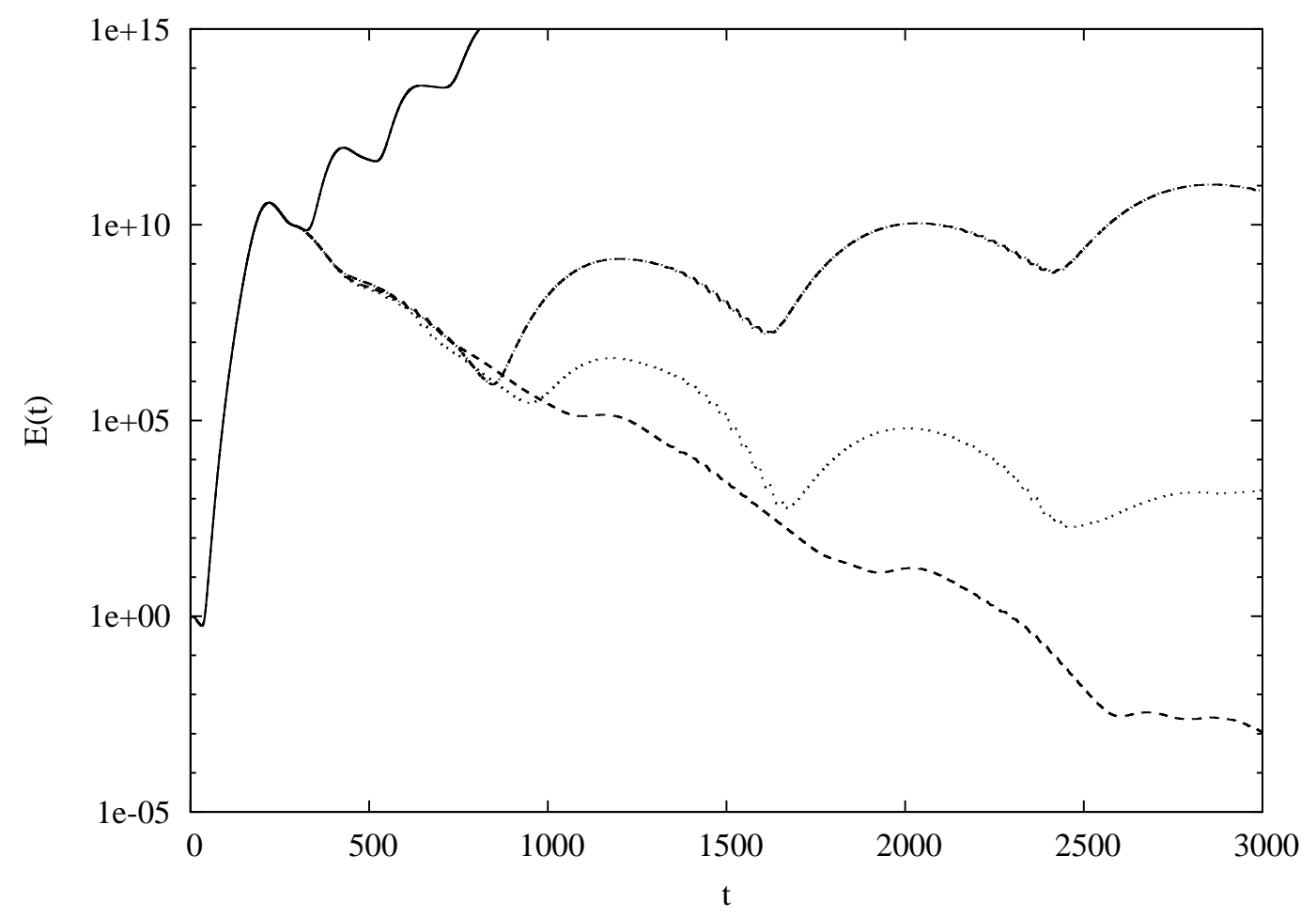

Fig. 8 Flow energy (normalized by $E(0))$ as function of time $(R e=590)$ : - uncontrolled case; - - estimator of dimension $n=400$ (double-projection); $\cdots$ estimator of dimension $n=100$ (double-projection); -. - : : estimator of dimension $n=100$ (bi-orthogonal projection).

and the solution vector can be integrated efficiently in time. Once the solution computed at time $t_{n+1}$, the skin-friction is measured up to second order in time the measure and at the next timestep $\mathbf{y}^{n+2} \approx 2 \mathbf{y}^{n+1}-\mathbf{y}^{n}$ enters into (32) (which is solved by second-order BDF too), providing the control at the new timestep.

\subsection{Control at $R e=590$}

First of all, the flow at $R e=590$ has been considered which exhibits only weakly unstable global instability modes. According to the above selection criterion, compensators with decreasing (real) dimensions $n=2 m$ have been considered, performing the double-projection as exposed in the previous section for the actuator $\mathbf{B}$ depicted in figure 3. As initial perturbation of the flow, a volume force of Gaussian-type, similar to the actuator structure but localized upstream of the bump near the entry of the flow domain, has been applied at $t=0$. The solid line in figure 8 shows the uncontrolled flow dynamics exhibiting a strong transient energy growth followed by the beating behavior. The associated dynamics has been analyzed in [9] in terms of the interaction between the global modes. Compensators with dimensions $n=400$ and $n=100$ have been considered (that is using $m=200$ and $m=50$ complex modes for the expansion, respectively). The results with control are depicted as the dashed and the dotted lines and a compensator with the fairly low dimension $n=100$ is seen to successfully control the flow dynamics. The control is seen to have no impact on the transient energy growth up to $t=200$. Indeed, the controller can start to act only after the initial disturbance (localized upstream) has reached the first sensor, the first skin-friction measurement starting at $x=60$.

Compensators obtained by conventional bi-orthogonal projection have also been computed and coupled to the plant. One result with $n=100$ is shown as the dashed-dotted line in figure 8 and the energy is shown to increase again after $t \approx 700$. Hence, in this case the alternative projection approach behaves favorably with regard to the dimension of the compensator. The uncontrolled flow structure is shown in figure 9 at $t=350,485,550$, illustrating the periodic (with period $T \approx 200$ ) regeneration of the 


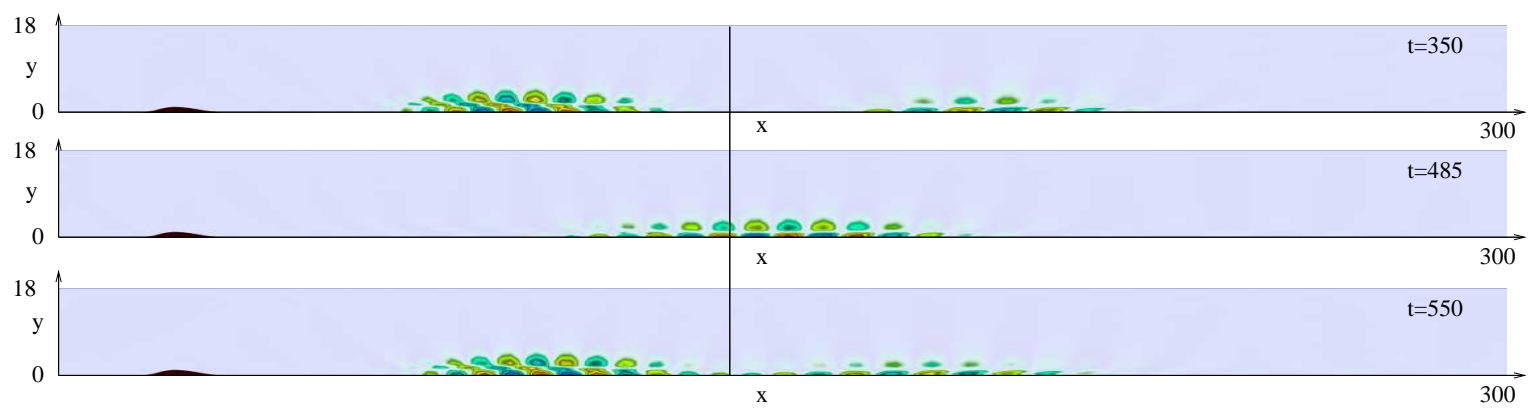

Fig. 9 Streamwise velocity component of the uncontrolled flow at $t=350,485,550$. The vertical line shows the location of the reattachment point of the basic flow recirculation bubble. The amplitude of the perturbation is normalized for each snapshot.

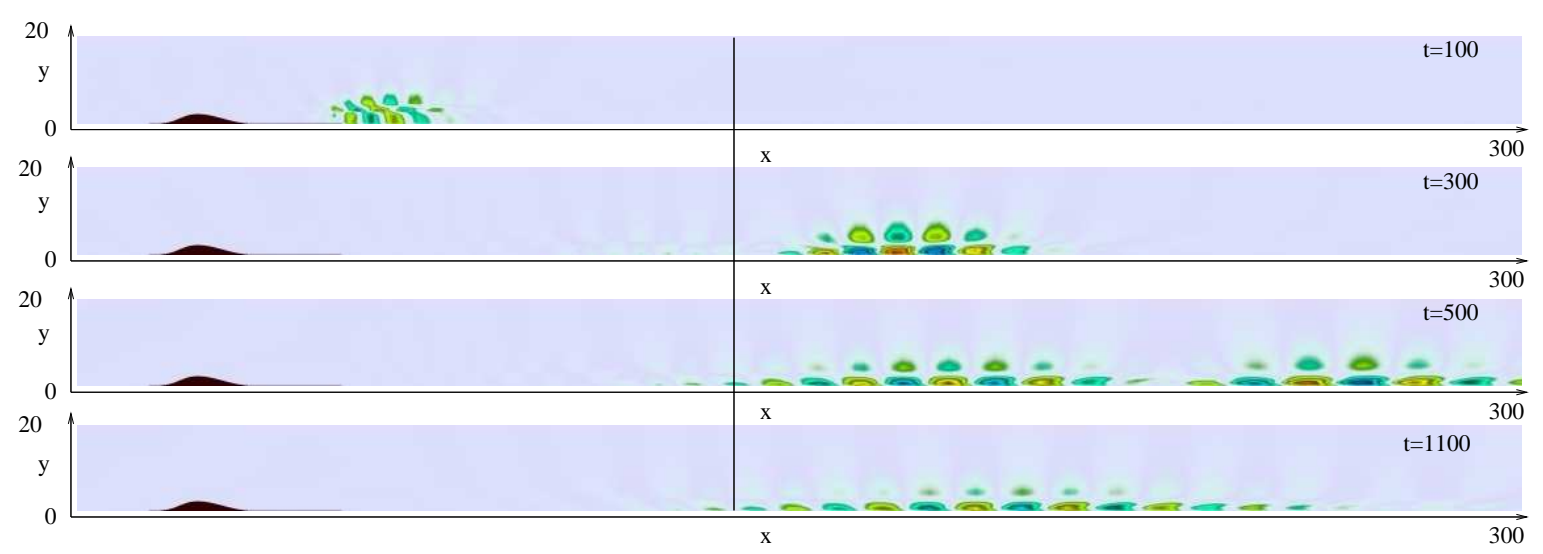

Fig. 10 Streamwise velocity component of the controlled flow at $t=100,300,500,1100$.

perturbation within the recirculation bubble. In figure 10 instantaneous flow structures are depicted when control is applied, with the estimator of dimension $n=400$ obtained by double-projection. Clearly the control prevents the regeneration of the perturbation which is washed downstream and eventually vanishes.

\subsection{Control at $R e=690$}

The flow control issue at the Reynolds number $R e=690$ is expected to be more involved, given the relatively high amplification rates of the modes as shown in figure 2. Again, the compensators for decreasing dimensions have been computed using the procedure outlined in section 3 . The flow dynamics is triggered by the same initial disturbance as previously at $R e=590$ and the same actuator is used in the plant. The uncontrolled total flow energy is shown as the solid line in figure 11. Again, there is a transient growth up to $t=200$, but now the subsequent global dynamics exhibits almost the same growth rate and the characteristic modulation due to the beating between the global modes is visible as well. Using the double-projection, a compensator with dimension $n=400$ is seen to control the flow and the energy is depicted as the dotted line in figure 11. The energy decrease is however seen to be relatively slow, in comparison with the flow case at $R e=590$. A compensator with dimension $n=300$ (with $m=150$ modes) has been considered as well and the result is shown as the dashed line in figure 11. This dimension is roughly the smallest possible to maintain a constant flow energy after the transient growth. Hence, comparing the results for both the Reynolds numbers $R e=590$ and $R e=690$, one may conclude that the required dimension of reliable compensators increases as the flow becomes more unstable away from threshold.

Again, the conventional bi-orthogonal projection of the actuator has been considered as well for the design of the compensator. As for $R e=590$, this projection procedure behaves less favorably, the 


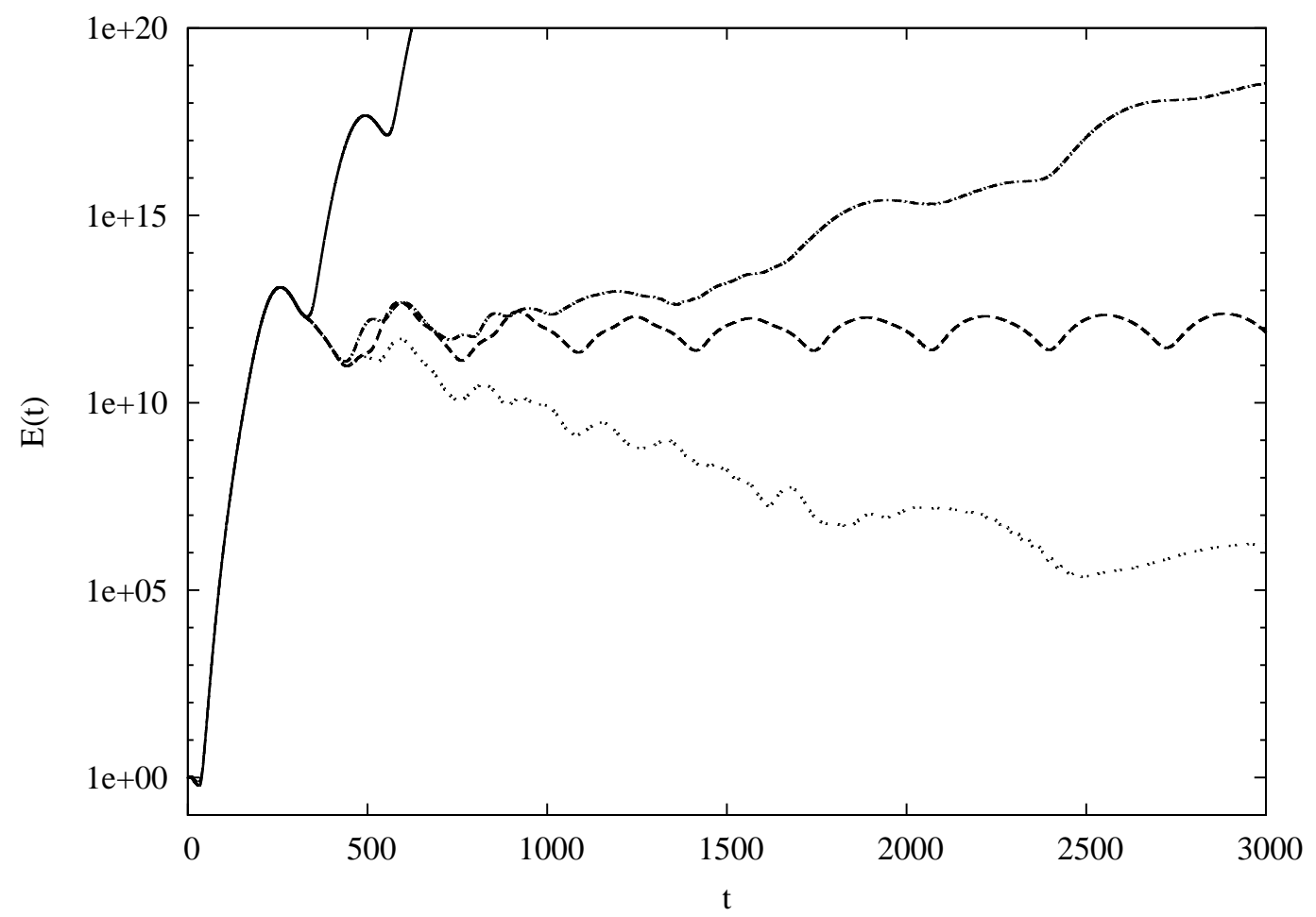

Fig. 11 Flow energy (normalized by $E(0))$ as function of time $(R e=690)$ : - - uncontrolled case; $\cdots$ estimator of dimension $n=400$ (double-projection); - - - estimator of dimension $n=300$ (double-projection); - . - . estimator of dimension $n=400$ (bi-orthogonal projection).

reduced controller with dimension $n=400$ being not sufficient to control the flow, as shown by the dashed-dotted line in figure 11.

\section{Concluding remarks}

Given the nowadays available computers and numerical methods, it has become customary to numerically analyze the instability behavior of open non-parallel flows in terms of eigenmodes depending on more than one space dimension. When adopting a dynamical system approach for flow control, the global modes appear naturally as a possible projection basis for model reduction. While these modes reproduce the instability physics, they may however not be efficient from the input-output viewpoint of system theory which uses the controllability and observability operators. In this latter case the actuator designed to control the flow as well as the sensor operator are part of the formulation, which however relies on the time-history of the dynamics.

For the separated boundary-layer flow along a bump geometry considered in the present work, the specific geometrical parameters and discretization used give rise to a large-scale numerical model for the flow perturbation dynamics. Readdressing the a priori model reduction using global modes, an alternative projection procedure, in comparison with conventional bi-orthogonal projection, is proposed. It uses the subset of stable modes to minimize the energy norm of the error between the real actuator and the reduced one. For the flow case considered, this procedure is shown to give rise to reliable and fairly low-dimensional controllers. Furthermore, this projection procedure is shown to perform better than the conventional approach based on the bi-orthogonality between direct and adjoint modes. Indeed, a compensator of dimension almost twice as high has to be taken in that case to achieve equivalent control.

The reliability of reduced order models based on global modes has been questioned recently, for instance in [5]. The present analysis provides indeed evidence, that the dimension of reliable controllers increases with the strength of the global flow instability. 


\section{References}

1. Åkervik, E., Hœpffner, J., Ehrenstein, U., Henningson, D.S.: Optimal growth, model reduction and control in a separated boundary-layer flow using global eigenmodes. J. Fluid Mech. 579, 305 - 314 (2007)

2. Alam, M., Sandham, N.: Direct numerical simulation of 'short' laminar separation bubbles with turbulent reattachment. J. Fluid Mech. 410, 1-28 (2000)

3. Bagheri, S., Brandt, L., Henningson, D.: Input-output analysis, model reduction and control of the flatplate boundary layer. J. Fluid Mech. 620, 263-298 (2009)

4. Barbagallo, A., Sipp, D., Jacquin, L., Schmid, P.: Control of an incompressible cavity flow using a reduced model based on global modes. AIAA paper 2008-3904, 5th AIAA Theoretical Fluid Mechanics Conference (2008)

5. Barbagallo, A., Sipp, D., Schmid, P.: Closed-loop control of an open cavity flow using reduced-order models. J. Fluid Mech., in press (2009)

6. Choi, H., Hinze, M., Kunisch, K.: Instantaneous control of backward-facing step flows. Appl. Numer. Maths 31, 133- 158 (1999)

7. Chomaz, J.M.: Global instabilities in spatially developing flows: non-normality and nonlinearity. Annu. Rev. Fluid Mech. 37, 357-392 (2005)

8. Ehrenstein, U., Gallaire, F.: On two-dimensional temporal modes in spatially evolving open flows: the flat-plate boundary layer. J. Fluid Mech. 536, 209-218 (2005)

9. Ehrenstein, U., Gallaire, F.: Two-dimensional global low-frequency oscillations in a separating boundarylayer flow. J. Fluid Mech. 614, 315-327 (2008)

10. Hœpffner, J., Chevalier, M., Bewley, T., Henningson, D.: State estimation in wall-bounded flow systems. part i: Laminar flows. J. Fluid Mech. 534, 263-294 (2005)

11. Ilak, M., Rowley, C.: Modeling of transitional channel flow using balanced proper orthogonal decomposition. Phys. Fluids 20, 034,103 (2008)

12. Kang, S., Choi, H.: Suboptimal feedback control of turbulent flow over a backward-facing step. J. Fluid Mech. 463, 201- 227 (2002)

13. Kim, J., Bewley, R.: A linear systems approach to flow control. Annu. Rev. Fluid Mech. 39, 383-417 (2007)

14. Kunkel, P., Mehrmann, V.: Differential-Alebraic Equations. EMS Textbooks in Mathematics (2006)

15. Marquillie, M., Ehrenstein, U.: On the onset of nonlinear oscillations in a separating boundary-layer flow. J. Fluid Mech. 490, 169-188 (2003)

16. Nayar, O., Ortega, U.: Computation of selected eigenvalues of generalized eigenvalue problems. J. Cmput. Phys. 108, 8-14 (1993)

17. Peyret, R.: Spectral Methods for Incompressible Flows. Springer (2002)

18. Rist, U., Maucher, U.: Investigations of time-growing instabilities in laminar separation bubbles. Eur. J. Mech. B/Fluids 21, 495- 509 (2002)

19. Rowley, C.: Model reduction for fluids using balanced proper orthogonal decomposition. Intl J. Bifurc. Chaos 15, 997-1013 (2005)

20. Trefethen, N., Embree, M.: Spectra and Pseudospectra: The Behaviour of Nonnormal Matrices and Operators. Princeton University Press (2005) 